\title{
Reliability of HR-pQCT Derived Cortical Bone Structural Parameters When Using Uncorrected Instead of Corrected Automatically Generated Endocortical Contours in a Cross-Sectional Study: The Maastricht Study
}

\author{
Ellis A. C. de Waard ${ }^{1,2}$. Cindy Sarodnik ${ }^{1,2}$ - Alexander Pennings ${ }^{1}$. Joost J. A. de Jong ${ }^{1,2} \cdot$ Hans H. C. M. Savelberg ${ }^{2,3}$. \\ Tineke A. van Geel ${ }^{2,4,5}$. Carla J. van der Kallen ${ }^{6,7}$. Coen D. A. Stehouwer ${ }^{6,7}$. Miranda T. Schram ${ }^{6,7,8}$. \\ Nicolaas Schaper ${ }^{4,6,7}$. Pieter C. Dagnelie, ${ }^{6,7}$ Piet P. M. M. Geusens ${ }^{4,9,10}$. Annemarie Koster ${ }^{4,11}$. \\ Bert van Rietbergen ${ }^{12}$. Joop P. W. van den Bergh ${ }^{2,9,10,13}$
}

Received: 26 January 2018 / Accepted: 22 March 2018 / Published online: 29 March 2018

(c) The Author(s) 2018

\begin{abstract}
Most HR-pQCT studies examining cortical bone use an automatically generated endocortical contour (AUTO), which is manually corrected if it visually deviates from the apparent endocortical margin (semi-automatic method, S-AUTO). This technique may be prone to operator-related variability and is time consuming. We examined whether the AUTO instead of the S-AUTO method can be used for cortical bone analysis. Fifty scans of the distal radius and tibia from participants of The Maastricht Study were evaluated with AUTO, and subsequently with S-AUTO by three independent operators. AUTO cortical bone parameters were compared to the average parameters obtained by the three operators (S-AUTOmean). All differences in mean cortical bone parameters between AUTO and S-AUTOmean were $<5 \%$, except for lower AUTO cortical porosity of the radius $(-16 \%)$ and tibia $(-6 \%)$, and cortical pore volume (Ct.Po.V) of the radius $(-7 \%)$. The ICC of S-AUTOmean and AUTO was $>0.90$ for all parameters, except for cortical pore diameter of the radius (0.79) and tibia (0.74) and Ct.Po.V of the tibia (0.89), without systematic errors on the Bland-Altman plots. The precision errors (RMS-CV\%) of the radius parameters between S-AUTOmean and AUTO were comparable to those between the individual operators, whereas the tibia RMS-CV\% between S-AUTOmean and AUTO were higher than those of the individual operators. Comparison of the three operators revealed clear inter-operator variability. This study suggests that the AUTO method can be used for cortical bone analysis in a cross-sectional study, but that the absolute values — particularly of the porosity-related parameters-will be lower.
\end{abstract}

Keywords High resolution peripheral quantitative computed tomography $\cdot$ Bone microarchitecture $\cdot$ Cortical bone analysis $\cdot$ Endocortical contour

\section{Introduction}

The geometry and density of the cortex of long bones are important determinants of bone strength. Since most of the bone mass lost with age is cortical, fractures at advanced age occur often at sites that mainly consist of cortical bone

Electronic supplementary material The online version of this article (https://doi.org/10.1007/s00223-018-0416-2) contains supplementary material, which is available to authorized users.

Ellis A. C. de Waard

e.dewaard@maastrichtuniversity.nl

Extended author information available on the last page of the article
[1-3]. Additionally, it has been shown that cortical porosity is a good predictor of bone strength [4-6]. High resolution peripheral quantitative computed tomography (HR-pQCT) is a non-invasive three-dimensional imaging modality that has the ability to measure volumetric bone mineral density (vBMD) and microarchitecture of the cortical and trabecular region [7]. Furthermore, HR-pQCT images can be used in micro-finite element analyses ( $\mu \mathrm{FEA}$ ) to calculate bone strength indices [8].

Identification of the cortical region on HR-pQCT images is challenging; the transition from endocortical to trabecular bone is gradual, so no single voxel identifies the end of the cortex and the beginning of the medullary canal with 
its trabecular content [9]. Currently, studies examining HRpQCT-derived cortical bone parameters mainly use a semiautomatic method (S-AUTO) provided by the manufacturer to distinguish the cortical region from the trabecular region. With this method, an endocortical contour is generated automatically first $[10,11]$, and the operator then manually modifies the generated contour when it visually deviates from the apparent endocortical margin. However, this method may be prone to operator-related variability and is time consuming (approximately $1 \mathrm{~h}$ per scan) [12]. This is particularly problematic in large cohort studies, where image analysis will be done by several operators and data from many participants need to be analyzed.

In an in vivo scan-rescan study by Kawalilak et al. it was shown that use of the uncorrected endocortical contour instead of S-AUTO contour resulted in the same repeatability of cortical bone parameters [12]. However, it is currently unknown whether the uncorrected contour can reliably be used for the assessment of cortical bone parameters in crosssectional studies. Additionally, the magnitude of differences in cortical parameters due to inter-operator variability in modification of the contour is unknown.

In this study, we examined whether the uncorrected automatically generated contour (AUTO) instead of the S-AUTO contour can be used for cortical bone analysis in an in vivo cross-sectional study. Therefore, cortical bone parameters were first obtained with the AUTO method, and then with endocortical contours that were corrected by three independent operators. The cortical bone parameters obtained with the AUTO method were compared to the average of the cortical bone parameters obtained with the S-AUTO method of the three independent operators (S-AUTOmean). Additionally, the cortical bone parameters obtained by the three independent operators were compared to each other. We hypothesized that the AUTO method can reliably be used for cortical bone analysis.

\section{Materials and Methods}

\section{Study Population and Design}

Data from The Maastricht Study, an observational prospective population-based cohort study, were used. The rationale and methodology of this study have been described previously [13]. In brief, the study focuses on the etiology, pathophysiology, complications, and comorbidities of type 2 diabetes (T2DM) and is characterized by an extensive phenotyping approach. Eligible for participation were all homedwelling individuals aged between 40 and 75 years and living in the southern part of the Netherlands. Participants were recruited through mass media campaigns and from the municipal registries and the regional Diabetes Patient
Registry via mailings. Recruitment was stratified according to known type 2 diabetes status, with an oversampling of individuals with T2DM for reasons of efficiency.

The present study includes cross-sectional data from a subset of 63 consecutive participants with normal glucose metabolism, who completed the baseline survey between September 2010 and June 2013 and returned to the research center between September 2015 and January 2016 for the HR-pQCT scan of the distal radius and tibia. Participants with a radius and/or tibia scan with severe or extreme motion artifacts (i.e., quality grade 4 or 5 [14], $n=11$ ), participants with a scan with an inadequate position of the reference line (reference line not on plateau of the distal radius or distal tibia $n=1$ ), and participants with extreme outliers on almost all cortical parameters ( $>2$ SD from mean, $n=1$ ) were excluded, resulting in a final study population of 50 participants. The study has been approved by the institutional medical ethical committee (NL31329.068.10) and the Minister of Health, Welfare and Sports of the Netherlands (Permit 131088-105234-PG). All participants gave written informed consent.

\section{HR-pQCT Imaging}

The non-dominant radius and ipsilateral tibia were scanned on a HR-pQCT scanner (XtremeCT; Scanco Medical AG, Brüttisellen, Switzerland) using the standard in vivo protocol as described in the literature [7, 15]. In case of a history of a fracture of the distal radius or tibia at that site, the contralateral site was scanned. The forearm and leg were immobilized in a carbon fiber cast. An anteroposterior scout projection of the scan site was acquired for positioning of the tomographic acquisition. A reference line was placed on the plateau of the distal radius or distal tibia. The scan started 9.5 and $22.5 \mathrm{~mm}$, for the radius and tibia respectively, from the reference line in the proximal direction and spanned $9.02 \mathrm{~mm}$ in length. Images were reconstructed using an isotropic voxel size of $82 \mu \mathrm{m}$, thus resulting in 110 consecutive slices. Total scan time was $2.8 \mathrm{~min}$, with each acquisition resulting in an effective dose of approximately $3 \mu \mathrm{Sv}$. All scans were graded once (operator 1) with regard to subject motion, and scans with quality grade 4 (severe motion artifacts) or 5 (extreme motion artifacts) were repeated once [14]. Only scans with quality grade 1 to 3 (no, minor or moderate motion artifacts) were used for subsequent image analysis [16].

\section{HR-pQCT Image Analysis}

All scans were evaluated using the standard patient evaluation protocol that was provided by the manufacturer and has been described previously in detail [17-19]. First, the periosteal contour was automatically derived and manually 
modified by operator 1 when contours visually deviated from the periosteal boundary [20]. The endocortical contour was automatically created using a series of automatic morphological operations to separate the trabecular and cortical volumes of interest [10], resulting in the uncorrected contour (AUTO method). Then, according to Burghardt et al. [10], when the contour visually deviated from the apparent endocortical margin, it was manually corrected (S-AUTO method). Correction of the AUTO contour was performed three times by three independent operators (operator 1 (OP1), operator 2 (OP2), and operator $3(\mathrm{OP} 3)$ ). All images were then analyzed using the advanced cortical evaluation protocol provided by the manufacturer $[10,11]$. All three operators underwent the same training for modification of the endocortical contour.

Evaluation of the cortical region resulted in the following parameters: cortical total volume $\left(\mathrm{Ct} . \mathrm{TV}, \mathrm{mm}^{3}\right)$, cortical bone volume $\left(\mathrm{Ct} . \mathrm{BV}, \mathrm{mm}^{3}\right)$, cortical thickness $(\mathrm{Ct} . \mathrm{Th}, \mathrm{mm})$, cortical vBMD (Ct.BMD, $\left.\mathrm{mgHA} / \mathrm{cm}^{3}\right)$, cortical pore volume (Ct.Po.V, $\mathrm{mm}^{3}$ ), cortical porosity (Ct.Po, \%), cortical pore diameter (Ct.Po.Dm, mm), and cortical area (Ct.Ar, $\mathrm{mm}^{2}$ ).

\section{Statistics}

All statistical analyses were performed using the Statistical Package for Social Sciences (version 22.0; IBM, Chicago, Illinois, USA). Mean and standard deviations for all cortical bone parameters were calculated using the S-AUTO contours of OP1, OP2, OP3, and (three times) the AUTO contour. All single evaluations with the AUTO method resulted in the same result; the average of the three AUTO evaluations (AUTOmean) is thus equal to AUTO. The average of the results obtained using the S-AUTO contours of OP1, OP2, and OP3, is referred to as S-AUTOmean. The mean difference in cortical bone parameters was calculated between S-AUTOmean and AUTO, and between all individual operators. A paired samples $t$ test was used to test for significant differences in mean cortical bone parameters between these pairs. Non-normally distributed variables were log transformed using the natural logarithm. The Pearson correlation coefficient $\odot$ and the intraclass correlation coefficient (ICC) were calculated to measure linear dependence and the level of agreement of the cortical bone parameters between S-AUTOmean and AUTO and between all individual operators. The precision error for all cortical bone parameters was calculated as the root mean square coefficient of variation (RMS-CV\%) of the three operators (OP1, OP2, and OP3), the pairs of operators (OP1 and OP2, OP1 and OP3, and OP2 and OP3), and the average of the semi-automatic method and the automatic method (S-AUTOmean and AUTO) [21]. Bland-Altman plots were provided to visualize agreement between S-AUTOmean and AUTO, and between the independent operators. The limits of agreement were calculated as the mean value $\pm 1.96 * \mathrm{SD}$. A $p$ value $<0.05$ was considered statistically significant.

\section{Results}

Data from 50 participants with normal glucose metabolism and a HR-pQCT scan of the distal radius and tibia with quality grade 1-3 were used for analysis. The mean age of the participants was $57.3 \pm 8.7$ year and $60 \%$ were women. Five (10.0\%) scans of the distal radius and $25(50.0 \%)$ scans of the distal tibia were graded as quality $1,29(58.0 \%)$ scans of the distal radius and $18(36.0 \%)$ scans of the distal tibia were graded as quality 2 , and $16(32.0 \%)$ scans of the distal radius and $7(14.0 \%)$ scans of the distal tibia were graded as quality 3 .

\section{Mean Cortical Bone Parameters}

The mean cortical bone parameters obtained by the individual operators and by AUTO and the differences in mean cortical parameters between S-AUTOmean and AUTO are shown in Table 1 (radius) and Table 3 (tibia). The differences in mean cortical parameters of the pairs of operators (OP1-OP2, OP1-OP3, and OP2-OP3) are shown in Table 2 (radius) and Table 4 (tibia).

The percentage difference in cortical bone parameters of the distal radius between S-AUTOmean-AUTO, and between the pairs of operators was generally low (5\% or less), except for Ct.Po.V and Ct.Po of the distal radius (Ct. Po.V: S-AUTOmean-AUTO 7.3\%, OP1-OP3 - 6.2\%, OP2-OP3 - 6.7\%; Ct.Po: S-AUTOmean-AUTO 16.1\%, OP1-OP3 - 14.0\%, OP2-OP3 - 14.2\%) and Ct.Po of the distal tibia (S-AUTOmean-AUTO 6.1\%). The mean cortical bone parameters obtained by AUTO were generally slightly lower than the parameters obtained by the independent operators.

\section{Correlation Coefficients}

Pearson's $r$ and the ICC of all bone parameters of the three operators (OP1-OP2-OP3) and of the two methods (S-AUTOmean-AUTO) are shown in Table 1 (radius) and Table 3 (tibia). Pearson's $r$ and the ICC of all bone parameters of the pairs of operators (OP1-OP2, OP1-OP3, and OP2-OP3) are shown in Table 2 (radius) and Table 4 (tibia).

The correlation coefficients for S-AUTOmean-AUTO and the pairs of operators were $>0.9$ for almost all cortical bone parameters of both the distal radius and tibia (except for $\log$ Ct.Po.Dm of the radius: S-AUTOmean-AUTO 0.89, OP1-OP3 0.88, OP2-OP3 0.87, and log Ct.Po.Dm of the tibia: S-AUTOmean-AUTO 0.80). The ICC of S-AUTOmean and AUTO was high $(>0.81)$ for almost all 


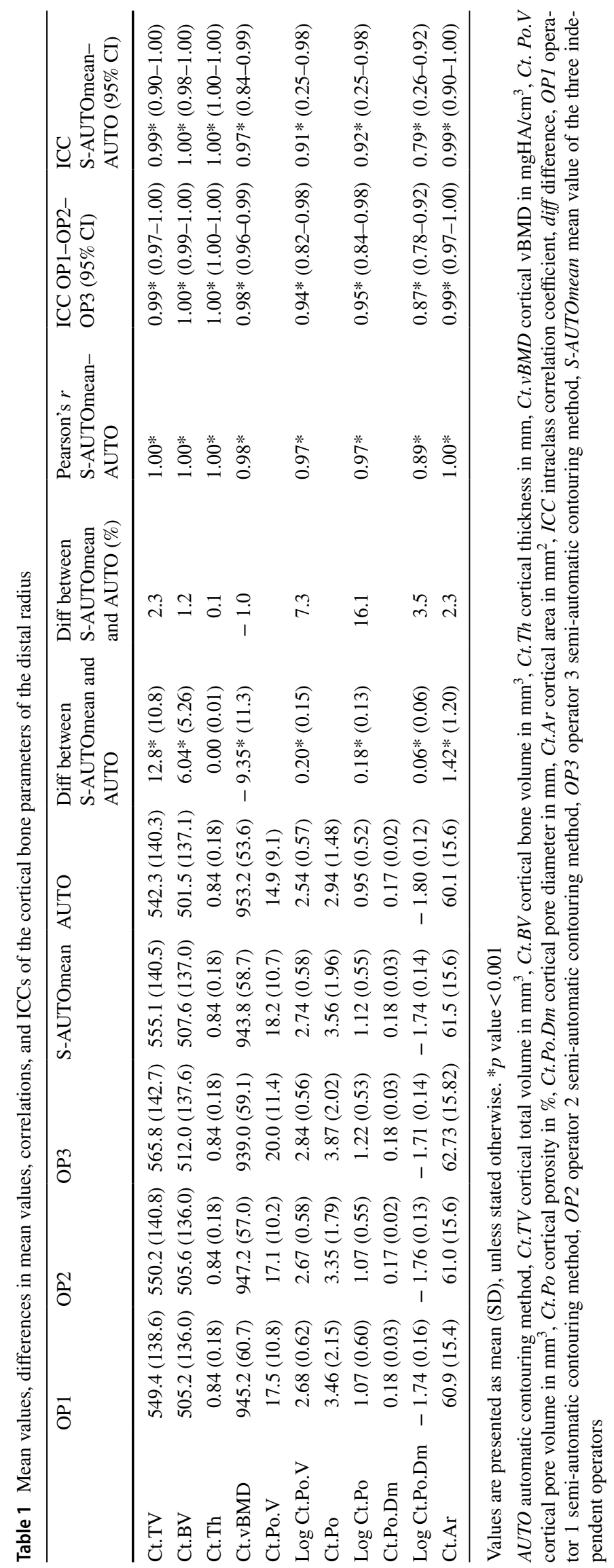




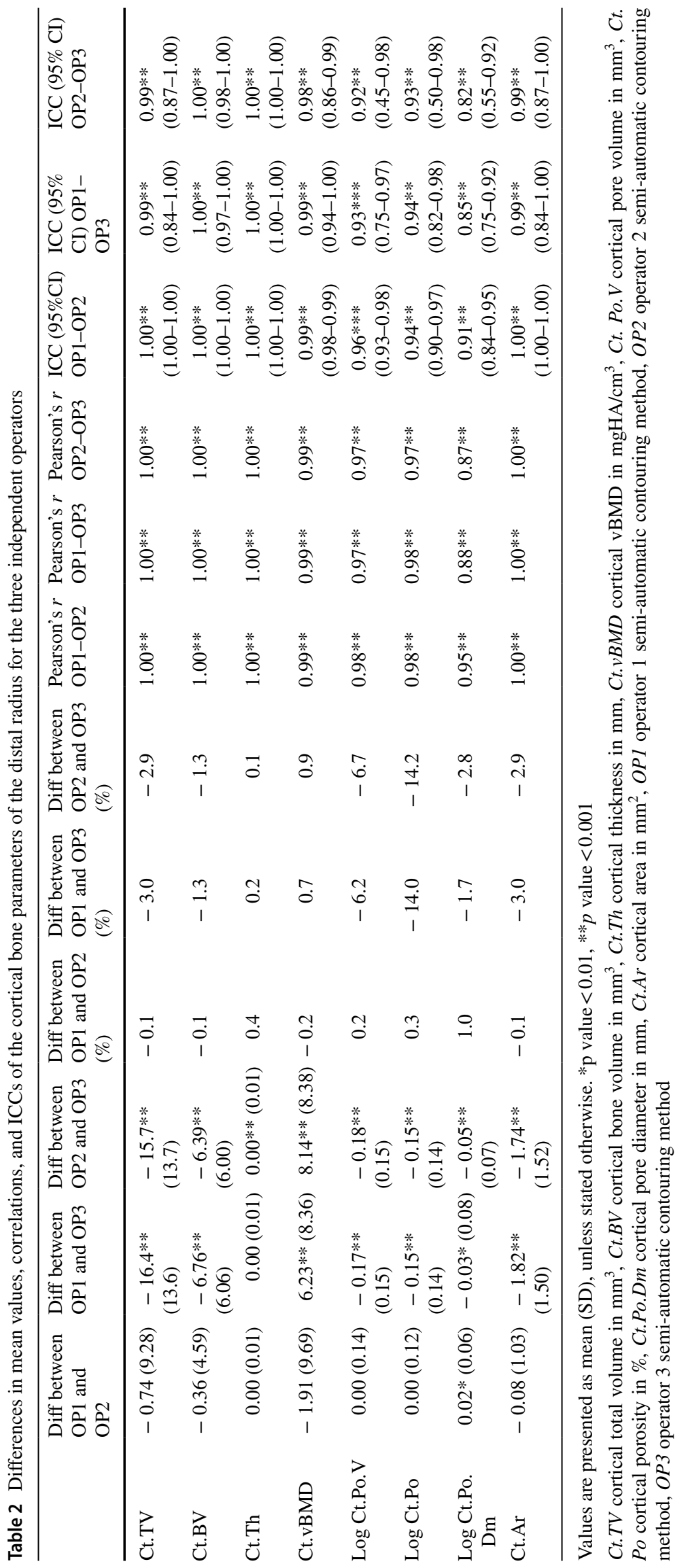




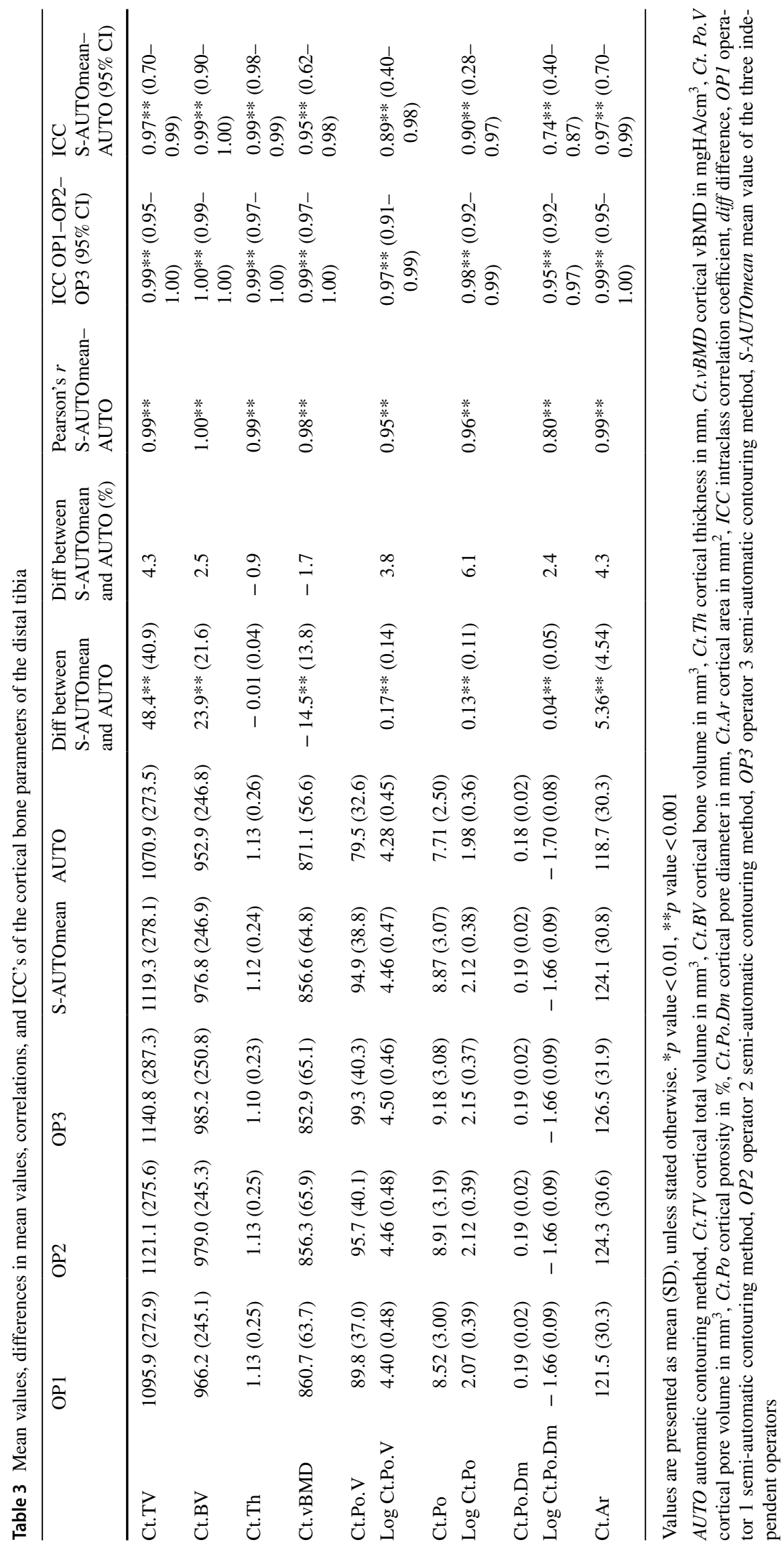




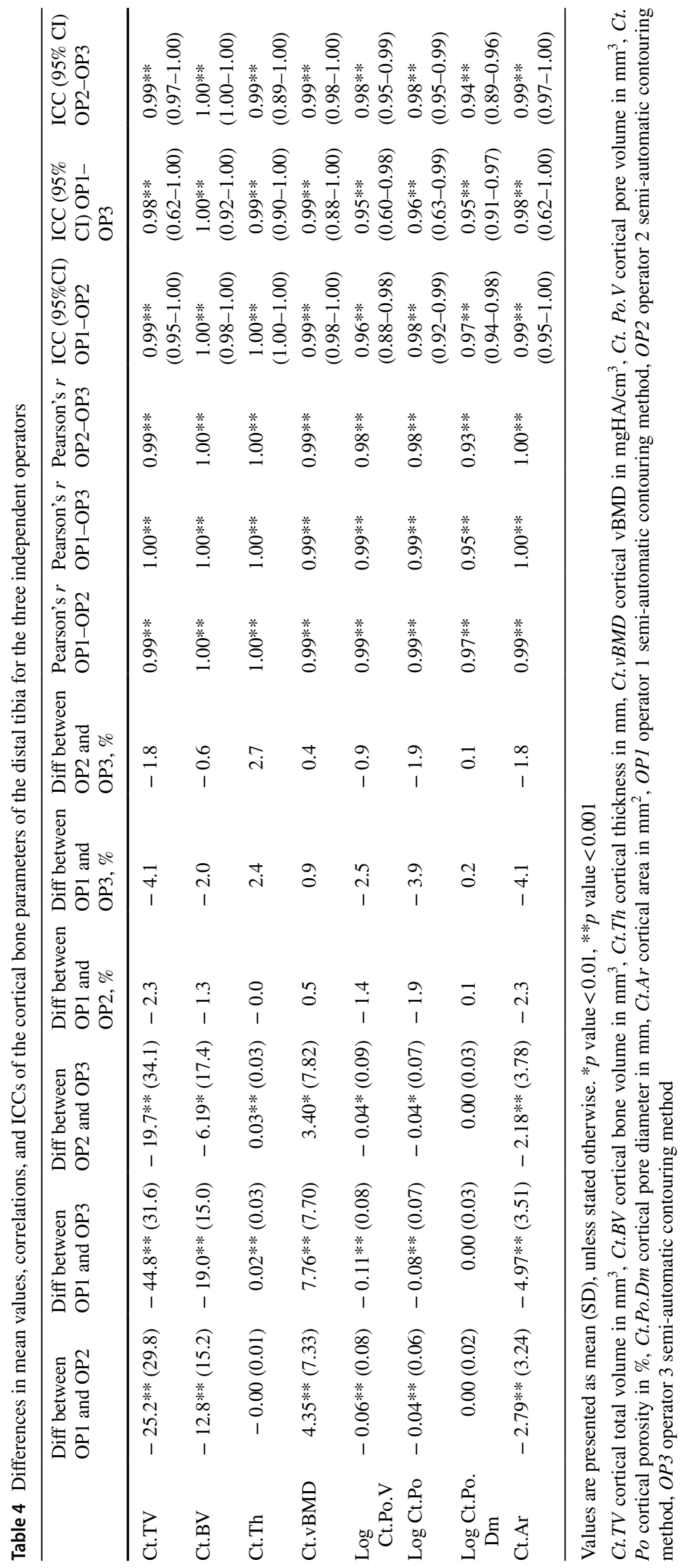


cortical bone parameters of the distal radius and tibia (except for $\log$ Ct.Po.Dm of the radius 0.79 and $\log$ Ct.Po.Dm of the tibia 0.74). The ICC of OP1-OP2-OP3 and the pairs of operators was $>0.81$ for all parameters of the distal radius and tibia.

\section{Precision Error}

The RMS-CV\% for all cortical bone parameters of both the distal radius and distal tibia are shown in Table 5 (OP1-OP2-OP3 and S-AUTOmean-AUTO) and Table 6 (pairs of operators). The precision error of the parameters Ct.TV, Ct.BV, Ct.Th, Ct.vBMD, and Ct.Ar was lower than the precision error of the porosity-related parameters (Ct.Po.V, Ct.Po, Ct.Po.Dm) for both the distal radius and tibia. The $95 \%$ confidence intervals of the precision errors of S-AUTOmean-AUTO of all cortical bone parameters of the distal radius had an overlap with the $95 \%$ confidence intervals of the precision errors of OP1-OP2-OP3, whereas the precision errors of S-AUTOmean-AUTO of the parameters of the distal radius were higher than those of OP1-OP2-OP3 (except for Ct.Th: OP1-OP2-OP3 1.26 (1.11-1.46), S-AUTOmean-AUTO 1.15 (0.96-1.43)).

\section{Bland-Altman Plots}

The Bland-Altman plots showed no systematic error in any of the plots of S-AUTOmean-AUTO (Fig. 1 (cortical porosity of the radius and tibia); Supplemental Figs. 1 (radius) and 2 (tibia)) and of the individual operators (Fig. 1 (cortical porosity of the radius and tibia); Supplemental Figs. 3 (radius) and 4 (tibia)). The 95\% confidence intervals were the widest in the plots of S-AUTOmean-AUTO, displaying more variability in error between S-AUTOmean and AUTO than between OP1 and OP2, OP1 and OP3, and OP2 and OP3. Additionally, use of the AUTO method instead of the S-AUTO method resulted in lower absolute values of the porosity-related parameters, while also clear variability in determined cortical porosity between the individual operators was observed (Fig. 1).

One outlier ( $>2$ SD from mean) was observed in the Bland-Altman plots of the distal radius of S-AUTOmean-AUTO and OP1-OP2 for the parameters Ct.TV, Ct.BV, Ct.Th, Ct.vBMD, and Ct.Ar. Slice 75 of this specific scan is shown in Fig. 2. Clear differences in the location of the endocortical contours of AUTO, OP2, and OP3 are visible when compared to the contour of OP1.
Table 5 Root mean square coefficient of variation (RMS-CV\%) of the cortical bone parameters of the distal radius and tibia

\begin{tabular}{lllll}
\hline & $\begin{array}{l}\text { Mean }(\mathrm{SD}) \\
\text { OP1/OP2/OP3 }\end{array}$ & $\begin{array}{l}\text { Mean (SD) } \\
\text { S-AUTOmean/AUTO }\end{array}$ & $\begin{array}{l}\text { RMS-CV\% (95\% CI) } \\
\text { OP1/OP2/OP3 }\end{array}$ & $\begin{array}{l}\text { RMS-CV\% (95\% CI) } \\
\text { S-AUTOmean/AUTO }\end{array}$ \\
\hline Radius & & & & \\
Ct.TV & $555.1(140.5)$ & $548.7(140.3)$ & $2.39(2.10-2.77)$ & $2.50(2.09-3.11)$ \\
Ct.BV & $507.6(137.0)$ & $504.6(137.0)$ & $1.21(1.06-1.41)$ & $1.39(1.16-1.73)$ \\
Ct.Th & $0.84(0.18)$ & $0.84(0.18)$ & $1.26(1.11-1.46)$ & $1.15(0.96-1.43)$ \\
Ct.vBMD & $943.8(58.7)$ & $948.5(55.9)$ & $0.85(0.74-0.99)$ & $1.15(0.97-1.44)$ \\
Ct.Po.V & $18.2(10.7)$ & $16.5(9.75)$ & $14.3(12.5-16.6)$ & $17.2(14.4-21.4)$ \\
Ct.Po & $3.56(1.96)$ & $3.25(1.69)$ & $12.9(11.3-15.0)$ & $15.3(12.8-19.0)$ \\
Ct.Po.Dm & $0.18(0.03)$ & $0.17(0.02)$ & $5.25(4.61-6.10)$ & $6.21(5.20-7.72)$ \\
Ct.Ar & $61.5(15.6)$ & $60.8(15.6)$ & $2.39(2.10-2.77)$ & $2.50(2.09-3.11)$ \\
Tibia & & & & \\
Ct.TV & $1119.3(278.1)$ & $1095.1(275.1)$ & $2.68(2.36-3.11)$ & $4.09(3.42-5.08)$ \\
Ct.BV & $976.8(246.9)$ & $964.9(246.6)$ & $1.47(1.29-1.71)$ & $2.44(2.04-3.03)$ \\
Ct.Th & $1.12(0.24)$ & $1.13(0.25)$ & $1.94(1.71-2.25)$ & $1.81(1.52-2.25)$ \\
Ct.vBMD & $856.6(64.8)$ & $863.9(60.4)$ & $0.80(0.71-0.93)$ & $1.73(1.44-2.14)$ \\
Ct.Po.V & $94.9(38.8)$ & $87.2(35.0)$ & $7.99(7.02-9.28)$ & $15.5(13.0-19.3)$ \\
Ct.Po & $8.87(3.07)$ & $8.29(2.75)$ & $6.19(5.44-7.19)$ & $12.1(10.1-15.0)$ \\
Ct.Po.Dm & $0.19(0.02)$ & $0.19(0.02)$ & $1.97(1.73-2.28)$ & $4.56(3.82-5.67)$ \\
Ct.Ar & $124.1(30.8)$ & $121.4(30.5)$ & $2.68(2.36-3.11)$ & $4.09(3.42-5.08)$ \\
\hline
\end{tabular}

$C t . T V$ cortical total volume in $\mathrm{mm}^{3}, C t . B V$ cortical bone volume in $\mathrm{mm}^{3}, C t$.Th cortical thickness in mm, $C t . v B M D$ cortical vBMD in $\mathrm{mgHA} / \mathrm{cm}^{3}, C t . P o . V$ cortical pore volume in $\mathrm{mm}^{3}, C t . P o$ cortical porosity in $\%$; Ct.Po.Dm cortical pore diameter in $\mathrm{mm}$; Ct.Ar cortical area in $\mathrm{mm}^{2}, O P 1$ operator 1 semi-automatic contouring method, $O P 2$ operator 2 semi-automatic contouring method, $O P 3$ operator 3 semi-automatic contouring method, S-AUTOmean mean value of the three independent operators, AUTO automatic contouring method 
Table 6 Root mean square coefficient of variation of the cortical bone parameters of the distal radius and tibia for the independent operators

\begin{tabular}{|c|c|c|c|c|c|c|}
\hline & $\begin{array}{l}\text { Mean (SD) } \\
\text { OP1-OP2 }\end{array}$ & $\begin{array}{l}\text { Mean (SD) } \\
\text { OP1-OP3 }\end{array}$ & $\begin{array}{l}\text { Mean (SD) } \\
\text { OP2-OP3 }\end{array}$ & $\begin{array}{l}\text { RMS-CV\% }(95 \% \text { CI }) \\
\text { OP1-OP2 }\end{array}$ & $\begin{array}{l}\text { RMS-CV\% }(95 \% \text { CI) } \\
\text { OP1-OP3 }\end{array}$ & $\begin{array}{l}\text { RMS-CV\% }(95 \% \text { CI }) \\
\text { OP2-OP3 }\end{array}$ \\
\hline \multicolumn{7}{|l|}{ Radius } \\
\hline Ct.TV & $549.8(139.6)$ & $557.6(140.5)$ & $558.0(141.6)$ & $1.48(1.24-1.84)$ & $2.66(2.22-3.30)$ & $2.77(2.32-3.44)$ \\
\hline Ct.BV & $505.4(136.7)$ & $508.6(136.8)$ & $508.8(137.5)$ & $0.91(0.76-1.13)$ & $1.29(1.08-1.61)$ & $1.38(1.15-1.71)$ \\
\hline Ct.Th & $0.84(0.18)$ & $0.84(0.18)$ & $0.84(0.18)$ & $1.51(1.26-1.88)$ & $1.10(0.92-1.37)$ & $1.09(0.91-1.36)$ \\
\hline Ct.vBMD & $946.2(58.7)$ & $942.1(59.8)$ & $943.1(57.9)$ & $0.81(0.68-1.01)$ & $0.82(0.69-1.02)$ & $0.92(0.77-1.14)$ \\
\hline Ct.Po.v & $17.3(10.4)$ & $18.7(11.0)$ & $18.5(10.7)$ & $9.48(7.93-11.8)$ & $15.51(13.0-19.3)$ & $15.9(13.3-19.8)$ \\
\hline Ct.Po & $3.40(1.95)$ & $3.67(2.07)$ & $3.61(1.89)$ & $8.41(7.04-10.5)$ & $14.2(11.9-17.6)$ & $14.4(12.1-17.9)$ \\
\hline Ct.Po.Dm & $0.18(0.03)$ & $0.18(0.03)$ & $0.18(0.02)$ & $4.03(3.37-5.01)$ & $5.65(4.73-7.02)$ & $5.82(4.87-7.24)$ \\
\hline Ct.Ar & $61.0(15.5)$ & $61.8(15.6)$ & $61.9(15.7)$ & $1.48(1.24-1.84)$ & $2.66(2.22-3.30)$ & $2.77(2.32-3.44)$ \\
\hline \multicolumn{7}{|l|}{ Tibia } \\
\hline Ct.TV & $1108.5(273.9)$ & $1118.4(279.8)$ & $1130.9(281.0)$ & $2.42(2.03-3.01)$ & $3.31(2.77-4.11)$ & $2.18(1.83-2.71)$ \\
\hline Ct.BV & $972.6(245.1)$ & $975.7(247.8)$ & $982.1(247.9)$ & $1.49(1.25-1.86)$ & $1.70(1.43-2.12)$ & $1.18(0.99-1.46)$ \\
\hline Ct.Th & $1.13(0.25)$ & $1.12(0.24)$ & $1.12(0.24)$ & $0.75(0.62-0.93)$ & $2.30(1.92-2.86)$ & $2.37(1.98-2.94)$ \\
\hline Ct.vBMD & $858.2(64.7)$ & $856.8(64.3)$ & $854.6(65.4)$ & $0.74(0.62-0.92)$ & $0.94(0.79-1.17)$ & $0.71(0.60-0.89)$ \\
\hline Ct.Po.v & $92.8(38.3)$ & $94.5(38.4)$ & $97.5(39.9)$ & $7.14(5.98-8.88)$ & $9.48(7.93-11.8)$ & $7.09(5.93-8.81)$ \\
\hline Ct.Po & $8.72(3.08)$ & $8.85(3.03)$ & $9.05(3.12)$ & $5.37(4.50-6.68)$ & 7.39 (6.18-9.19) & $5.59(4.67-6.94)$ \\
\hline Ct.Po.Dm & $0.19(0.02)$ & $0.19(0.02)$ & $0.19(0.02)$ & $1.68(1.41-2.09)$ & $1.97(1.65-2.45)$ & $2.18(1.83-2.71)$ \\
\hline Ct.Ar & $122.9(30.4)$ & $124.0(31.0)$ & $125.4(31.2)$ & $2.42(2.03-3.01)$ & $3.31(2.77-4.11)$ & $2.18(1.83-2.72)$ \\
\hline
\end{tabular}

$C t . T V$ cortical total volume in $\mathrm{mm}^{3}, C t . B V$ cortical bone volume in $\mathrm{mm}^{3}, C t . T h$ cortical thickness in mm, Ct.vBMD cortical vBMD in mgHA/ $\mathrm{cm}^{3}, C t$. Po.V cortical pore volume in $\mathrm{mm}^{3}$, Ct.Po cortical porosity in \%,Ct.Po.Dm cortical pore diameter in mm, Ct.Ar cortical area in mm ${ }^{2}$, $O P 1$ operator 1 semi-automatic contouring method, $O P 2$ operator 2 semi-automatic contouring method, $O P 3$ operator 3 semi-automatic contouring method

\section{Discussion}

In this study, we evaluated whether the AUTO contour instead of the S-AUTO contour could be used for cortical bone analysis in a cross-sectional study. Therefore, the (average) results obtained with the AUTO method were compared to the average results of the S-AUTO method. Additionally, variability in results as obtained with the different S-AUTO contours was examined.

The smallest differences in mean cortical bone parameters, the highest ICCs and lowest precision error were found between independent operators. Additionally, the precision error of the three operators was generally lower than the precision error of S-AUTOmean and AUTO. This indicates that correction of the contours, even when the scans are analyzed by several independent operators, introduces a smaller error than the use of the uncorrected, automatically generated contours. However, the mean differences in cortical bone parameters between S-AUTOmean and AUTO were highly comparable to the differences between OP1 and OP3. The absolute level of agreement between S-AUTOmean and AUTO was high and the Bland-Altman plots of the differences between S-AUTOmean and AUTO in cortical parameters showed no systematic error.

The precision errors of the cortical bone parameters of the distal radius of OP1-OP3 and of OP2-OP3 were generally comparable to those of S-AUTOmean-AUTO. In contrast, the precision errors of the cortical bone parameters of the distal tibia of S-AUTOmean and AUTO were higher than the precision error of the individual operators, particularly for the porosity-related parameters. Compared to precision errors for cortical bone parameters obtained by a short-term repositioning study that used S-AUTO [10], the precision errors of S-AUTOmean and AUTO were higher, particularly those of Ct.Po.V and Ct.Po of the distal tibia. In contrast, the error introduced by not correcting the endocortical contour to the precision was comparable to the error introduced by variability in positioning of the reference line for the parameters Ct.BMD and Ct.Th, but not for Ct.Po [22]. Additionally, the precision error of S-AUTOmean and AUTO for Ct.Po and Ct.Po.Dm of the distal radius is comparable to the precision error of a donor specimen that was scanned and evaluated at 9 different sites [23]. Although it is known that motion of the subject has a large influence on the precision error of densitometry and trabecular microarchitectural parameters [14], the influence of motion of the subject on cortical bone parameters has not been examined. Thus, the AUTO method seems to introduce about the same error to the precision error as variability in the position of the reference line and as multicenter scanning.

Although all operators underwent the same training, clear differences between the endocortical contours of 

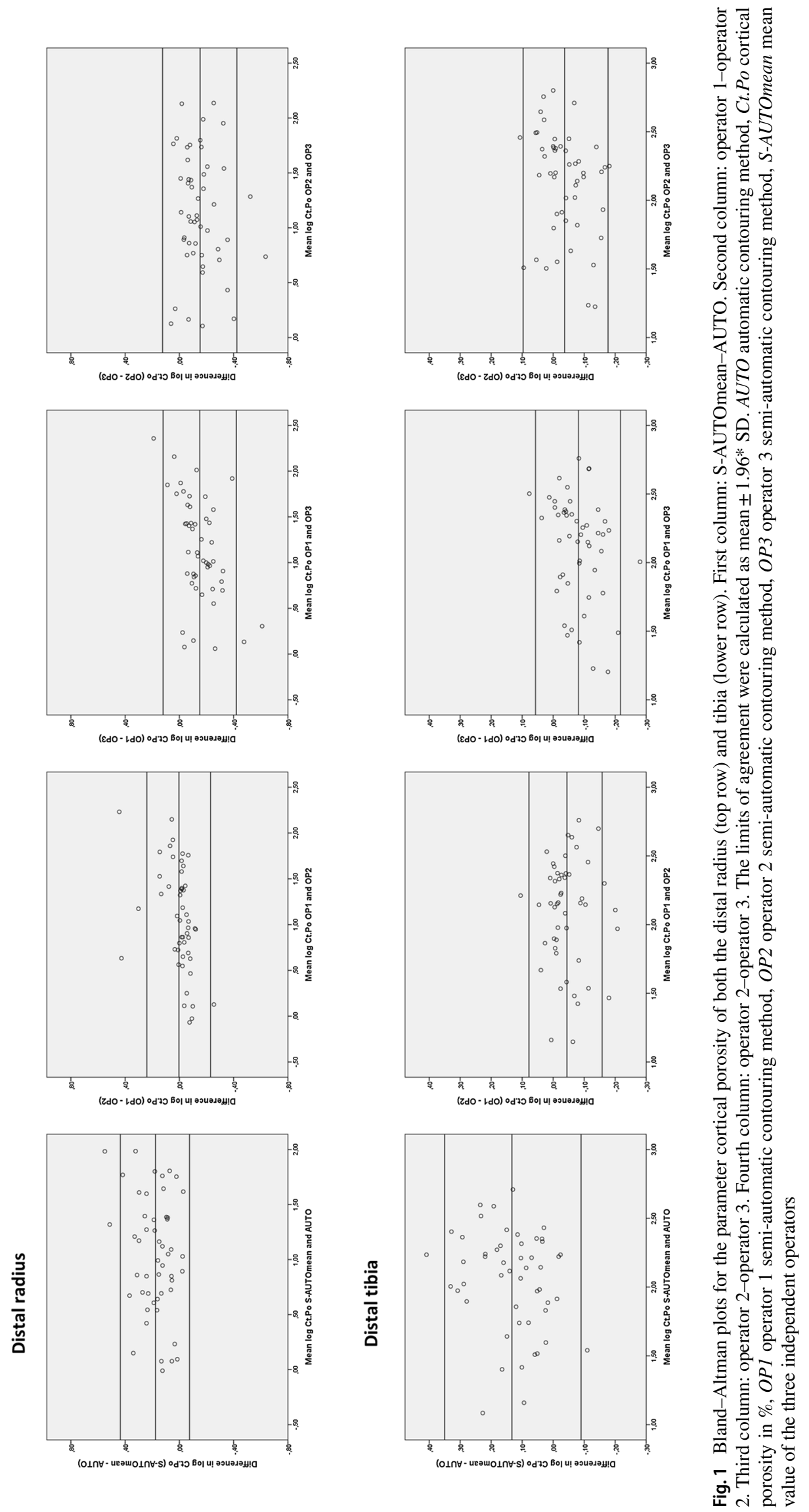

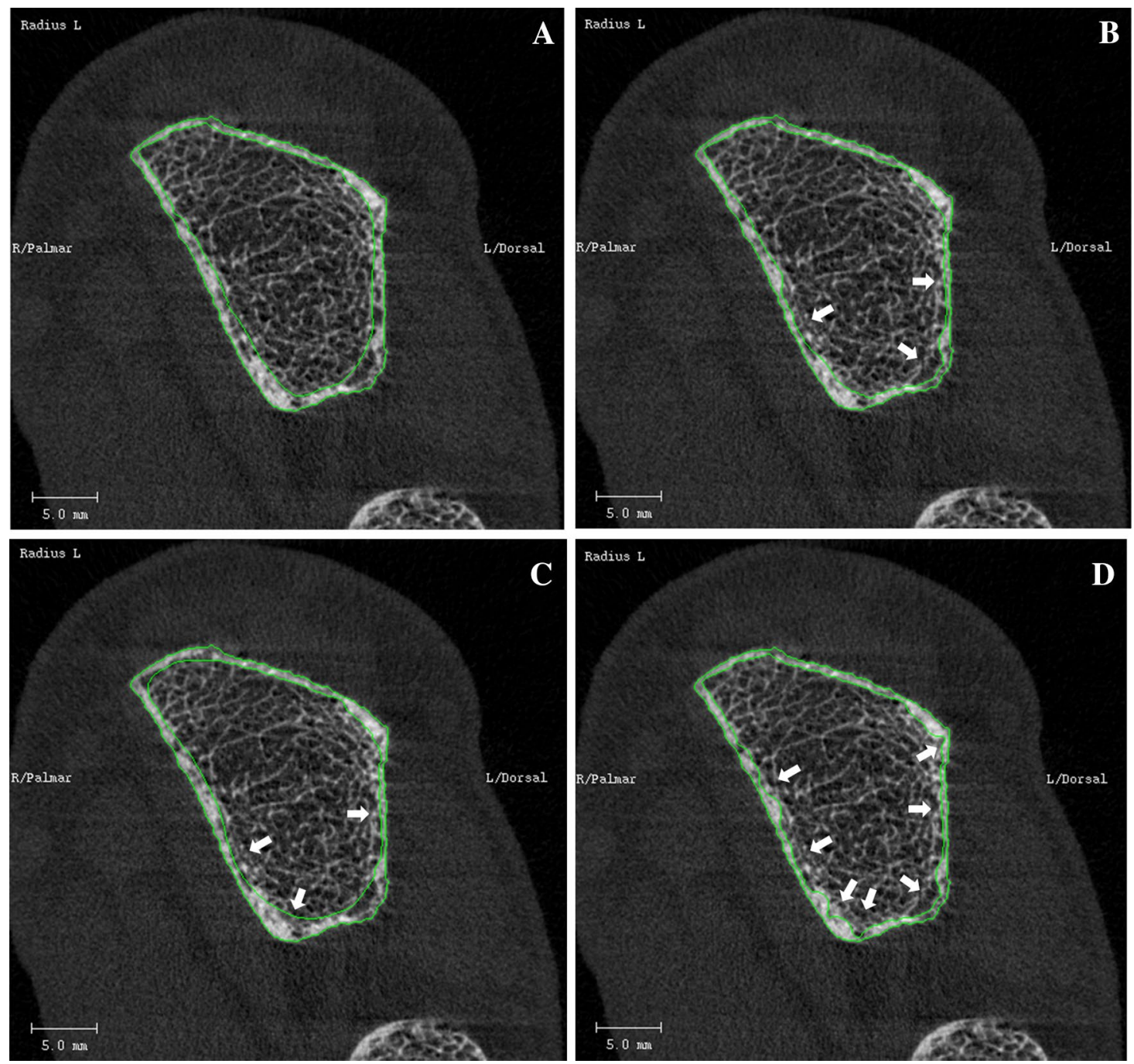

Fig. 2 Slice 75 of an HR-pQCT scan of the distal radius, as modified by operator 1 (a), operator 2 (b), operator 3 (c), and the automatic method (d). The arrows indicate the differences in the endocortical contour of operator 2 , operator 3 , and the automatic method, com-

the three operators were visible (Fig. 2). The explanation for this difference is probably the gradual transition from cortical to trabecular bone, which makes identification of the endocortical border challenging [1,9]. As a result of the presence of this transitional zone, each operator 'sees' his or her own truth and modifies the AUTO contour in a slightly different way, thereby including variable parts of the transitional zone. Currently, there are no studies published that compare different endocortical contours with histology (the golden standard), and it is thus currently unknown which contour marks the endocortical border correctly. Since the porosity of the trabecular compartment is much higher than the porosity of the cortical compartment, variability in inclusion of the transitional zone will influence the observed cortical bone parameters pared to operator 1 . The presented scan is the outlier in the BlandAltman plots of the distal radius of S-AUTOmean-AUTO and OP1OP2 for the parameters Ct.TV, Ct.BV, Ct.Th, Ct.vBMD, and Ct.Ar

and will lead to an increased RMS-CV\%, particularly of the porosity-related parameters. As shown in Fig. 2, the endocortical contour created by AUTO was closer to the periosteal border than the contour created by the independent operators, and thus included a smaller part of the transitional zone. As a result, use of AUTO results in lower absolute values of cortical porosity when compared to using the S-AUTO method (Fig. 1). This is in agreement with the study by Kawalilak et al., who also showed that the S-AUTO method resulted in a larger trabecularized cortex compared to the AUTO method [12]. Studies using AUTO contours instead of correct contours should therefore take into account that this will lead to lower absolute values of all parameters, except for the cortical vBMD which will be higher. 
Limitations of our study include the generalizability; the mean age of our study population was 58 years, and therefore most of the included women will be postmenopausal. It may be expected that the AUTO method will also be reliable in premenopausal women because the endocortical border is often better recognizable in younger women [1]. Additionally, a study by Kawalilak et al. showed that the reproducibility of cortical bone parameters when the AUTO contour is used is better in premenopausal than in postmenopausal women [12]. In contrast, recognition of the endocortical border is more difficult in older individuals, which may lead to problems with both the AUTO and S-AUTP method. Future studies are warranted to examine the validity of the use of the AUTO method in both younger and older study populations. A second limitation of our study is the lack of scan-rescan data. We were therefore not able to determine the short- and/or long-term reproducibility of the uncorrected and corrected contours. However, a previous study showed a high reproducibility of both the AUTO method and the corrected contours [12]. Third, the study was not designed for comparing the two methods for a clinical outcome such as fracture prediction or treatment of osteoporosis. Future studies are warranted to examine whether the AUTO contour can also be used in studies with clinical outcomes. Fourth, for the examination of the cortical compartment, we used the method provided by the manufacturer. Therefore, all the results in this study are therefore only valid for this method, and cannot be extrapolated to other algorithms such as the commercially available $\operatorname{StrAx} \odot$ software [24]. Finally, the reference line for the HR-pQCT scans was placed at a fixed reference point, which resulted in the scanning of the same region in every participant. However, bone morphology at that region differs between individual patients, where a higher amount of cortical bone will be present in participants with relatively short extremities. Therefore, recent studies suggest scanning at a percentage distance of the total length of the bone [25, 26].

In conclusion, the S-AUTO method resulted in better reliability of the cortical bone parameters than the AUTO method in a cross-sectional study. However, it was shown that correction of the AUTO contour by different operators resulted in clear variability in cortical parameters. Additionally, the percent differences in cortical parameters, the ICCs, and the precision errors of the radius between the uncorrected automatically generated contour and the corrected contour were highly comparable to the errors observed between independent operators and no systematic error was observed in the Bland-Altman plots. Therefore, we believe that the AUTO contour can be used for cortical bone analysis in a cross-sectional study, although it should be taken into account that use of the AUTO instead of the S-AUTO method will result in lower absolute values of particularly the porosity-related parameters. The lower cortical parameters with AUTO and the variability in parameters between the individual operators can be explained by variable inclusion of the transitional zone.

Author contributions EACdW, PPMMG, AK, BvR, and JPWvdB designed the study and prepared the first draft of the paper. EACdW is guarantor. EACdW, CS, and AP contributed to the experimental work. EACdW, JJAdJ, PPPMG, AK, BvR, and JPWvdB were responsible for statistical analysis of the data. All authors revised the paper critically for intellectual content and approved the final version. All authors agree to be accountable for the work and to ensure that any questions relating to the accuracy and integrity of the paper are investigated and properly resolved.

Funding This study was supported by the European Regional Development Fund via OP-Zuid, the Province of Limburg, the Dutch Ministry of Economic Affairs (Grant 310.041), Stichting De Weijerhorst (Maastricht, the Netherlands), the Pearl String Initiative Diabetes (Amsterdam, the Netherlands), the Cardiovascular Center (CVC, Maastricht, the Netherlands), Cardiovascular Research Institute Maastricht (CARIM, Maastricht, the Netherlands), School for Public Health and Primary Care (CAPHRI, Maastricht, the Netherlands), School for Nutrition, Toxicology and Metabolism (NUTRIM, Maastricht, the Netherlands), Stichting Annadal (Maastricht, the Netherlands), Health Foundation Limburg (Maastricht, the Netherlands) and by unrestricted grants from Janssen-Cilag B.V. (Tilburg, the Netherlands), Novo Nordisk Farma B.V. (Alphen aan den Rijn, the Netherlands), and SanofiAventis Netherlands B.V. (Gouda, the Netherlands).

\section{Compliance with Ethical Standards}

Conflict of interest Bert van Rietbergen is consultant for Scanco Medical AG. Piet Geusens received Grants from Abbott, BMS, Amgen, UCB, MSD, Novartis, Pfizer, Roche, and Will Pharma and personal fees from Amgen and UCB outside the submitted work. Ellis AC de Waard, Cindy Sarodnik, Alexander Pennings, Joost JA de Jong, Hans HCM Savelberg, Tineke A van Geel, Carla J van der Kallen, Coen DA Stehouwer, Miranda T Schram, Nicolaas Schaper, Pieter C Dagnelie, Annemarie Koster and Joop PW van den Bergh declare that they have no conflict of interest.

Human and Animal Rights and Informed Consent All procedures performed in studies involving human participants were in accordance with the ethical standards of the institutional and/or national research committee and with the 1964 Helsinki declaration and its later amendments or comparable ethical standards. The study has been approved by the institutional medical ethical committee (NL31329.068.10) and the Minister of Health, Welfare and Sports of the Netherlands (Permit 131088-105234-PG). All participants gave written informed consent.

Open Access This article is distributed under the terms of the Creative Commons Attribution 4.0 International License (http://creativeco mmons.org/licenses/by/4.0/), which permits unrestricted use, distribution, and reproduction in any medium, provided you give appropriate credit to the original author(s) and the source, provide a link to the Creative Commons license, and indicate if changes were made. 


\section{References}

1. Zebaze RM, Ghasem-Zadeh A, Bohte A et al (2010) Intracortical remodelling and porosity in the distal radius and post-mortem femurs of women: a cross-sectional study. Lancet 375(9727):1729-1736. https://doi.org/10.1016/s0140 $-6736(10) 60320-0$

2. Riggs BL, Wahner HW, Dunn WL, Mazess RB, Offord KP, Melton LJ 3rd (1981) Differential changes in bone mineral density of the appendicular and axial skeleton with aging: relationship to spinal osteoporosis. J Clin Invest 67(2):328-335. https://doi. org/10.1172/jci110039

3. Kanis JA, Johnell O, Oden A, Dawson A, De Laet C, Jonsson B (2001) Ten year probabilities of osteoporotic fractures according to BMD and diagnostic thresholds. Osteoporos Int 12(12):989995. https://doi.org/10.1007/s001980170006

4. Bala Y, Zebaze R, Seeman E (2015) Role of cortical bone in bone fragility. Curr Opin Rheumatol 27(4):406-413

5. Wachter NJ, Augat P, Krischak GD, Mentzel M, Kinzl L, Claes L (2001) Prediction of cortical bone porosity in vitro by microcomputed tomography. Calcif Tissue Int 68(1):38-42. https://doi. org/10.1007/bf02685001

6. Augat P, Schorlemmer S (2006) The role of cortical bone and its microstructure in bone strength. Age Ageing. https://doi. org/10.1093/ageing/afl081

7. Boutroy S, Bouxsein ML, Munoz F, Delmas PD (2005) In vivo assessment of trabecular bone microarchitecture by high-resolution peripheral quantitative computed tomography. J Clin Endocrinol Metab 90(12):6508-6515. https://doi.org/10.1210/ jc.2005-1258

8. MacNeil JA, Boyd SK (2007) Accuracy of high-resolution peripheral quantitative computed tomography for measurement of bone quality. Med Eng Phys 29(10):1096-1105. https://doi. org/10.1016/j.medengphy.2006.11.002

9. Zebaze R, Seeman E (2015) Cortical bone: a challenging geography. J Bone Miner Res 30(1):24-29. https://doi.org/10.1002/ jbmr.2419

10. Burghardt AJ, Buie HR, Laib A, Majumdar S, Boyd SK (2010) Reproducibility of direct quantitative measures of cortical bone microarchitecture of the distal radius and tibia by HR-pQCT. Bone 47(3):519-528. https://doi.org/10.1016/j.bone.2010.05.034

11. Nishiyama KK, Macdonald HM, Buie HR, Hanley DA, Boyd SK (2010) Postmenopausal women with osteopenia have higher cortical porosity and thinner cortices at the distal radius and tibia than women with normal aBMD: an in vivo HR-pQCT study. J Bone Miner Res 25(4):882-890. https://doi.org/10.1359/jbmr.091020

12. Kawalilak CE, Johnston JD, Cooper DM, Olszynski WP, Kontulainen SA (2016) Role of endocortical contouring methods on precision of HR-pQCT-derived cortical micro-architecture in postmenopausal women and young adults. Osteoporos Int 27(2):789796. https://doi.org/10.1007/s00198-015-3262-3

13. Schram MT, Sep SJ, van der Kallen CJ et al (2014) The Maastricht study: an extensive phenotyping study on determinants of type 2 diabetes, its complications and its comorbidities. Eur J Epidemiol 29(6):439-451. https://doi.org/10.1007/s10654-014-9889-0

14. Pialat JB, Burghardt AJ, Sode M, Link TM, Majumdar S (2012) Visual grading of motion induced image degradation in high resolution peripheral computed tomography: impact of image quality on measures of bone density and micro-architecture. Bone 50(1):111-118. https://doi.org/10.1016/j.bone.2011.10.003

15. Khosla S, Riggs BL, Atkinson EJ et al (2006) Effects of sex and age on bone microstructure at the ultradistal radius: a population-based noninvasive in vivo assessment. J Bone Miner Res 21(1):124-131. https://doi.org/10.1359/jbmr.050916

16. Pauchard Y, Liphardt AM, Macdonald HM, Hanley DA, Boyd SK (2012) Quality control for bone quality parameters affected by subject motion in high-resolution peripheral quantitative computed tomography. Bone 50(6):1304-1310. https://doi. org/10.1016/j.bone.2012.03.003

17. Laib A, Hauselmann HJ, Ruegsegger P (1998) In vivo high resolution 3D-QCT of the human forearm. Technol Health Care 6(5-6):329-337

18. Laib A, Ruegsegger $P$ (1999) Comparison of structure extraction methods for in vivo trabecular bone measurements. Comput Med Imaging Graph 23(2):69-74

19. Hildebrand T, Rüegsegger $P$ (1997) A new method for the model-independent assessment of thickness in three-dimensional images. J Microsc 185(1):67-75. https://doi.org/10.104 6/j.1365-2818.1997.1340694.x

20. Buie HR, Campbell GM, Klinck RJ, MacNeil JA, Boyd SK (2007) Automatic segmentation of cortical and trabecular compartments based on a dual threshold technique for in vivo micro-CT bone analysis. Bone 41(4):505-515. https://doi.org/10.1016/j. bone.2007.07.007

21. Gluer CC, Blake G, Lu Y, Blunt BA, Jergas M, Genant HK (1995) Accurate assessment of precision errors: how to measure the reproducibility of bone densitometry techniques. Osteoporos Int 5(4):262-270

22. Bonaretti S, Vilayphiou N, Chan CM et al (2017) Operator variability in scan positioning is a major component of HR-pQCT precision error and is reduced by standardized training. Osteoporos Int 28(1):245-257. https://doi.org/10.1007/s00198-016-3705-5

23. Burghardt AJ, Pialat JB, Kazakia GJ et al (2013) Multicenter precision of cortical and trabecular bone quality measures assessed by high-resolution peripheral quantitative computed tomography. J Bone Miner Res 28(3):524-536. https://doi.org/10.1002/ jbmr. 1795

24. Zebaze R, Ghasem-Zadeh A, Mbala A, Seeman E (2013) A new method of segmentation of compact-appearing, transitional and trabecular compartments and quantification of cortical porosity from high resolution peripheral quantitative computed tomographic images. Bone 54(1):8-20. https://doi.org/10.1016/j. bone.2013.01.007

25. Bonaretti S, Majumdat S, Lang TF, Khosla S, Burghardt AJ (2017) The comparability of HR-pQCT bone measurements is improved by scanning anatomically standardized regions. Osteoporos Int 28(7):2115-2128. https://doi.org/10.1007/s00198-017-4010-7

26. Shanbhogue VV, Hansen S, Halekoh U, Brixen K (2015) Use of relative vs fixed offset distance to define region of interest at the distal radius and tibia in high-resolution peripheral quantitative computed tomography. J Clin Densitom 18(2):217-225. https:// doi.org/10.1016/j.jocd.2014.12.002 


\section{Affiliations}

Ellis A. C. de Waard ${ }^{1,2}$ (1) . Cindy Sarodnik ${ }^{1,2} \cdot$ Alexander Pennings $^{1} \cdot$ Joost J. A. de Jong ${ }^{1,2} \cdot$ Hans H. C. M. Savelberg ${ }^{2,3}$. Tineke A. van Geel ${ }^{2,4,5}$. Carla J. van der Kallen ${ }^{6,7}$. Coen D. A. Stehouwer6,7 . Miranda T. Schram ${ }^{6,7,8}$.

Nicolaas Schaper ${ }^{4,6,7}$. Pieter C. Dagnelie, ${ }^{6,7}$ Piet P. M. M. Geusens ${ }^{4,9,10}$. Annemarie Koster ${ }^{4,11}$. Bert van Rietbergen ${ }^{12}$. Joop P. W. van den Bergh ${ }^{2,9,10,13}$

1 Department of Internal Medicine, Subdivision of Rheumatology, Maastricht University, P.O. Box 616, 6200 MD Maastricht, The Netherlands

2 NUTRIM School for Nutrition and Translational Research in Metabolism, Maastricht University, Maastricht, The Netherlands

3 Department of Human Movement Science, Maastricht University, Maastricht, The Netherlands

4 CAPHRI Care and Public Health Research Institute, Maastricht University, Maastricht, The Netherlands

5 Department of Family Medicine, Maastricht University, Maastricht, The Netherlands

6 Department of Internal Medicine, Maastricht University Medical Center, Maastricht, The Netherlands

7 CARIM School for Cardiovascular Diseases, Maastricht University, Maastricht, The Netherlands
8 Heart and Vascular Center, Maastricht University Medical Centre, Maastricht, The Netherlands

9 Department of Internal Medicine, Subdivision of Rheumatology, Maastricht University Medical Centre, Maastricht, The Netherlands

10 Biomedical Research Institute, University of Hasselt, Hasselt, Belgium

11 Department of Social Medicine, Maastricht University, Maastricht, The Netherlands

12 Faculty of Biomedical Engineering, Eindhoven University of Technology, Eindhoven, The Netherlands

13 Department of Internal Medicine, Subdivision of Endocrinology, VieCuri Medical Center, Venlo, The Netherlands 